QUADERNS DE FILOSOFIA VOL. II NÚM. I (2015): 79-9I

ISSN: 234 I-I 4 I 4

DOI: I0.7203/QFIA.2.I.4554

Diego SÁnchez MecA

UNED

\title{
La transformación nietzscheana del pensamiento según Deleuze
}

Recibido: 12/1/15. Aceptado: 17/2/15

Resumen: Deleuze ve en Nietzsche a uno de los filósofos que con mayor eficacia crítica contradice la concepción clásica del pensamiento como búsqueda de la verdad y como ejercicio de amor a la verdad. Este artículo analiza el significado que para Deleuze tiene la oposición de Nietzsche a la tradición filosófica, que considera que el pensamiento quiere y ama la verdad de la que nos vemos alejados por la acción de fuerzas extrañas al pensamiento (procedentes del cuerpo, de las pasiones, de los intereses, etc.). De este modo se muestra de qué modo esas fuerzas que intervienen en el pensamiento remiten el pensamiento mismo a las fuerzas que le subyacen en cuanto pensamiento.

\begin{abstract}
Nietzsche is considered by Deleuze one of the philosophers who most critical efficacy contradicts the classical conception of thought as search for truth and exercise of love for truth. This article analyzes the significance for Deleuze of Nietzsche's opposition to the philosophical tradition in that sense. The believe that thinking wants and loves the truth is also the believe that we are away from the action of foreign forces thought (from the body, passions, interest, etc.). Thus Deleuze shows how these forces involved in thought itself refers to the forces underlying it as thought.
\end{abstract}

Palabras clave: Deleuze, Nietzsche, pensamiento, verdad, cuerpo.

Keywords: Deleuze, Nietzsche, thought, truth, body. 
A L IGUAL QUE el de Foucault, el pensamiento de Deleuze creció a la sombra del de Nietzsche y la cuestión del nihilismo afirmativo, o sea, centrado en la noción de filosofía como crítica. ${ }^{1}$ En este sentido, el de Deleuze es un pensamiento típicamente moderno, pues, en realidad, esta noción de filosofía como crítica radical es la noción que anima todo el pathos de la filosofía moderna, de la Ilustración, del proceso de secularización y del deseo de liberación que subyace e impulsa este pathos. Deleuze ve en Nietzsche a uno de los filósofos que con mayor eficacia crítica contradice la concepción clásica, metafísica y platónica del pensamiento como búsqueda de la verdad y como ejercicio de amor a la verdad. Para esta tradición, nos vemos alejados de la verdad por la interferencia de fuerzas extrañas al pensamiento que proceden del cuerpo, de las pasiones, de los intereses, por lo que es necesario cierto ascetismo unido a un método lógico o dialéctico argumentativo para pensar bien, o sea, para pensar lo verdadero. Lo que Nietzsche objeta a esta concepción del pensamiento es que no tiene en cuenta ni es consciente de las fuerzas reales que verdaderamente intervienen y guían el pensamiento, ni refiere el pensamiento a las fuerzas reales que supone en cuanto pensamiento.

En su obra Proust y los signos (I964), elaborada por Deleuze casi simultáneamente a su espléndido estudio dedicado a Nietzsche, Nietzsche y la filosofía, destaca hasta qué punto las sensaciones de dolor y de placer, las variaciones de intensidad de estas sensaciones, el efecto de un encuentro fortuito con imágenes o con signos son capaces de despertar, de estimular y de poner en funcionamiento el pensamiento y la reflexión. Lo cual demuestra que no es cierto que todas esas fuerzas "extrańas al pensamiento" (entre comillas) nos aparten de la verdad, como siempre han defendido racionalistas y metafísicos, sino que son ellas los determinantes esenciales para su búsqueda y su revelación. En la obra de Proust es frecuente encontrar situaciones en las que la verdad se ofrece antes de dejarse descubrir. Porque muchos de los personajes de las novelas proustianas traicionan un secreto, un secreto celosamente guardado, a través de un gesto que no viene a cuento, o una palabra fuera de lugar, o un comportamiento que contrasta con los hábitos cotidianos de esa persona. Esto lleva a Deleuze a concluir que a la idea racionalista cartesiana de método, Proust opone la doble idea de la coacción y del azar. La verdad depende de un encuentro con algo que nos obliga a pensar y a buscar la verdad. Por ello, el azar de los encuentros y la presión de la coacción son los dos temas fundamentales de la novelística de Proust. Y lo que constituye el objeto de un encuentro, o sea, lo que ejerce sobre nosotros esa coacción, es, para Deleuze, el signo.

\footnotetext{
${ }^{1}$ Este es el texto de la conferencia pronunciada en la Jornada Internacional "Perspectivas de investigación sobre Nietzsche", celebrada en la Facultad de Filosofía de la Universitat de València el 30 de octubre de 2014, y coordinada por Pietro Gori y Paolo Stellino.
} 
Merece la pena intentar ver, en la medida de lo posible, este nuevo estatuto atribuido por Deleuze al pensamiento filosófico, fijándonos en la filiación de los planteamientos de Deleuze respecto de Nietzsche, y remitiendo sus categorías centrales a los conceptos nietzscheanos más propiamente distintivos de su obra. Por ejemplo, en relación con este estatuto mismo del pensamiento, podemos recordar cómo la idea central que subyace a la genealogía nietzscheana es justamente ésta, o sea, que el pensamiento nunca piensa por sí mismo, ni encuentra por sí mismo la verdad, sino que está siempre condicionado y determinado por relaciones de fuerzas que se establecen con la mediación del cuerpo. Por tanto, la pregunta filosófica “¿Qué es lo verdadero?” debería quedar sustituida por estas otras: “¿Quién busca la verdad?”, “¿Qué quiere o qué pretende quien afirma tal o cual cosa como verdad?” Pues pensar, afirma Nietzsche, no es el ejercicio natural, libre y consciente de una facultad, sino que depende siempre de las fuerzas que se apoderan del pensamiento y hacen de él un acontecimiento extraordinario e imprevisible.

Esta es la oposición de la genealogía nietzscheana a la imagen dogmática del pensamiento, propia de la tradición filosófica, a partir de la cual reivindica todo un nuevo concepto de filosofía como ejercicio práctico de valoración y de interpretación, que lo que busca, ante todo, es poder determinar el sentido y el valor que producidos por esas fuerzas y por esas relaciones de fuerzas que despiertan y guían al pensamiento: "Juzgar es nuestra creencia más antigua, nuestro más habitual tener por-verdadero o por-no-verdadero. En el juicio se encuentra nuestra creencia más antigua, en todo juzgar hay un tener por verdadero o un tener por no verdadero, un afirmar o negar, una certeza de que algo es así y no de otra manera, una creencia de que allí se ha 'conocido' efectivamente - ¿qué es lo que se cree verdadero en todo juicio?" (NieTzSCHE 2007, 84). Y este es, como se sabe, el programa filosófico que Nietzsche atribuye como propio y original de los filósofos presocráticos, que comprendían la filosofía ligada a la vida, y que se diluye cuando la filosofía se convierte en pura teoría y en metafísica por obra, sobre todo, de Sócrates y de Platón.

Pues bien, esta contraposición nietzscheana entre la pura razón metafísica y el pensamiento determinado por las fuerzas de la vida y del cuerpo, aspira a producir, también en el pensamiento de Deleuze, una nueva imagen del pensamiento que recupere aquella relación presocrática entre pensamiento y vida, en la forma justamente de la coacción que la vida ejerce sobre el pensamiento. Por ello, este pensamiento no puede concebir la verdad ya sino apoyada en una tipología pluralista de las fuerzas y, por tanto, desde las categorías de sentido y valor, y no desde la distinción entre lo verdadero y lo falso; ni puede reconducir la verdad a la representación, que implica el reconocimiento de lo existente en sí, o sea, el conformismo con lo que hay, sino que tiene que 
reformularla a partir de nociones nuevas como son la de signo, la de diferencia o la de repetición. A Deleuze le atrae, pues, esta perspectiva crítica de Nietzsche y, decidido a desarrollar las ambiciosas propuestas que representa, emprende un interesantísimo análisis de la obra nietzscheana que, a partir de 1962 (año en que se publica Nietzsche et la philosophie), sigue incansable a lo largo de toda su trayectoria intelectual, en busca de respuestas a los distintos y decisivos problemas que se plantean.

Una de las principales líneas de este desarrollo es la que se refiere al análisis de la lógica de la identidad, y que discurre a partir de la contraposición entre el tradicional pensamiento de lo idéntico frente a un pensamiento sintético como pensamiento de lo absolutamente diferente. Deleuze lo expresa así en alusión a la idea nietzscheana del eterno retorno: "Lo dispar es el último elemento de la repetición, que se opone a la identidad de la representación. Es por tal motivo que el circulo del eterno retorno, el de la diferencia y de la repetición (que deshace el de lo idéntico y lo contradictorio), es un circulo tortuoso, que no dice lo Mismo más que de lo que difiere" (Deleuze 2002, 102-3). Hay en este punto de partida deleuziano una clara influencia de Bergson a la que conviene aludir para que se entienda mejor el problema. Una de las cosas por las que Bergson llama la atención de Deleuze la constituye su denuncia de la complicidad del concepto tradicional de pensamiento con el predominio exclusivo de las representaciones espaciales, lo que entrañaba la práctica imposibilidad de pensar en términos de duración. Por ello, lo que hace Bergson es contraponer a la metafísica de la sustancia su concepción del flujo o impulso vital desde el que comprende el devenir de la vida como energía creadora. $Y$ es este devenir el que debe sustituir, según él, a las viejas esencias estáticas de la metafísica. El método bergsoniano para captar este devenir es la intuición, una operación que permite distinguir lo complejo que nos ofrece la experiencia y apreciar las diferencias de naturaleza sin confundirlas con meras diferencias de grado. Es frente a este método, dice Deleuze, frente al que la estupidez del pensamiento tradicional habría proclamado que "todo es equivalente", que todo es lo mismo, o sea, ser, el ser.

La dificultad que entraña comprender bien esto puede verse, tal vez, superada recurriendo a un determinado estudio de las obras de arte que nos permita esbozar las líneas de fuerza de los problemas fundamentales. Concretamente, hay que fijarse en las repeticiones que son inherentes a las obras de arte, y concentrarnos en la noción de estilo, una noción, por cierto, muy nietzscheana. Porque concentrándonos en esta noción de estilo se puede tomar conciencia con facilidad de lo que significa concretamente esa unidad fundamental del sentido como potencia de la diferencia que hace retornar, en cada una de las obras de arte distintas entre sí, una y otra vez, las mismas relaciones 
de fuerza. Y, para ilustrar esto, Deleuze cita a Marcel Proust cuando, hablando de esta misma relación de la diferencia y la repetición, se refiere a la sonata de Vinteuil (una composición musical de estructura análoga al Bolero de Ravel), y comentaba que tal o cual frase de la sonata retornaba la misma una y otra vez, pero cada vez cambiada sobre un ritmo o un acompañamiento diferente; la misma y, sin embargo, otra, como retornan las cosas en la vida.

Es decir, lo que retorna aquí, una y otra vez, no es una identidad, una idea platónica pura y siempre igual a sí misma, sino algo concreto, unos sonidos, unas intensidades sonoras, unas separaciones rítmicas, una distribución en el tiempo de las diferencias intensivas no contradictorias, diferentes "modalidades", etcétera. Pero, al mismo tiempo, lo que se repite en cada retornar es el todo, o sea, el mundo de esas intensidades que se diferencian sin cesar. Por tanto, la diferencia es indisociable de estos dos tipos distintos de repetición, la de lo diferente y la de lo mismo, que se sintetizan precisamente en el estilo. Así lo dice Deleuze con estas palabras: "Existe una gran diferencia entre las dos repeticiones, la material y la espiritual. Una es repetición de instantes o de elementos sucesivos independientes; la otra, una repetición del Todo, en niveles diversos coexistentes (como decía Leibniz, 'siempre y en todo lugar lo mismo', salvo en los grados de perfección!). Por tal motivo, las dos repeticiones mantienen una relación muy diferente con la diferencia misma. La diferencia es sustraída a una, en la medida en que los elementos o instantes se contraen en un presente viviente. Está incluida en la otra, en la medida en que el Todo comprende la diferencia entre sus niveles. Una está desnuda, la otra vestida; una es de las partes, la otra del todo; una de sucesión, la otra de coexistencia; una actual, la otra virtual, una horizontal, la otra vertical" (Deleuze 2002, 139).

Y es que la repetición, así comprendida, nada cambia en el objeto que repite, sino que cambia algo en el espíritu que la contempla: esta fue la célebre tesis, primero de Heráclito, y luego de Hume, que nos conduce al corazón mismo del problema: ¿Cómo la repetición puede cambiar algo en el caso o elemento que se repite, puesto que implica siempre una clara independencia de cada presentación? La regla de la discontinuidad o de la instantaneidad en la repetición se formula, por tanto, en los siguientes términos: nada aparece sin que otra cosa haya desaparecido. Este era el estado del río heraclíteo como fluir de momentos. ¿Cómo puede decirse entonces que "el segundo" momento del río, o "el tercero" son lo mismo, el mismo río si la repetición se deshace a medida que se hace? Esto solo tiene una contestación, a saber, que la repetición carece de un en-sí. Lo único que cambia es algo en el espíritu de quien contempla el río. Tal es la esencia de la modificación. Por su parte, Hume toma como ejemplo una repetición casuística, del tipo $\mathrm{AB}, \mathrm{AB}, \mathrm{AB}$, o sea, si $\mathrm{A}$ entonces $\mathrm{B}$. 
Cada caso, cada secuencia objetiva $\mathrm{AB}$ es independiente de la otra. Por tanto, la repetición no cambia nada en el objeto, en el estado de las cosas AB. Sí se produce, sin embargo, una transformación en el espíritu del que contempla. Cuando A aparece, yo espero ya la aparición de B. ¿Es esto el para sí de la repetición como subjetividad necesaria que debe entrar necesariamente en su constitución? La paradoja de la repetición ¿̇acaso no es que no puede hablarse de repetición sino mediante la diferencia o el cambio que introduce en el espíritu que la contempla?

Podemos, pues, preguntarnos a partir de esta reflexión sobre la repetición: ¿Qué es, entonces, lo que en las obras de arte marca la diferencia, la esencia que las diversifica, pero que, a la vez, se repite en ellas como tal, idéntica a sí misma como potencia de diferenciación? Deleuze responde: el estilo. El estilo, que no es la propiedad exclusiva de una obra concreta, sino que es la esencia misma. Pero una esencia que se revela en lo que los cuerpos muestran, es decir, no como algo que se descubre en cuanto verdad en el sentido platónico del término, sino como algo que se manifiesta en cuanto mero simulacro. En suma, la revelación de la esencia se produce en la obra de arte a través de una unidad del signo, de la esencia y del sentido como diferencia en acto, como estilo que se repite y diversifica multiplicando los signos inmateriales, los encuentros y las rupturas. Esto significa que el estilo no es más que una pura apariencia. Y esta es la razón de por qué el estilo funciona como verdadero proceso de individuación, porque el arte nos ofrece la imagen de la verdadera unidad en cuanto unidad de un signo y de su sentido espiritual.

En conclusión, el retorno de lo semejante no es más que un efecto de la repetición considerada como potencia de la diferencia. Siempre es en el mismo movimiento donde la repetición comprende la diferencia. Pues la repetición no se entiende aquí en ningún caso como repetición de lo mismo, sino siempre de lo diferente como tal, teniendo la diferencia en sí misma por objeto la repetición. Éste sería propiamente el ser del devenir, y aquí tenemos que fijar nuestra atención. Porque en relación a las connotaciones de innovación que esta concepción de la repetición supone frente a las concepciones metafísicas del pensamiento tradicional, conviene atender a dos consecuencias que este planteamiento tiene de enorme trascendencia crítica. Por un lado, el eterno retorno de la diferencia como ser del devenir representa el cuestionamiento más extremo de la representación clásica de la duración, del tiempo y de la relación fundamental que mantienen con la memoria, así como con la separación habitual de pasado, presente y futuro. De modo que con la concepción del eterno retorno se deja de considerar ya el tiempo a partir de las nociones estáticas y abstractas de presente, pasado y futuro, y se aborda en su dinamismo concreto considerando las nebulosas, los mundos y los vectores que se expresan 
en él bajo la forma de líneas distintas. Estas líneas constituyen series, de modo que podemos afirmar que el tiempo mismo es serial. Por otro lado, el eterno retorno arruina la filosofía clásica de la representación porque arruina todo el pensamiento del origen y del modelo, ya que el eterno retorno, tomado en su sentido estricto, significa que cada cosa solo existe retornando, copia de una infinidad de copias que no dejan subsistir ningún original ni ningún origen. Lo que es o retorna no tiene ninguna identidad previa y constituida: las cosas se reducen a la diferencia que las separan y a todas las diferencias implicadas en ellas y por las cuales pasan. Esta es la razón de por qué se dice que el eterno retorno es paródico, porque califica lo que hace ser (y retornar) como siendo un puro simulacro. La idea misma (la esencia) no es más que el efecto de una combinación finita de simulacros cuyas combinaciones son infinitas.

Esta idea del simulacro ha tenido y tiene una productividad filosófica muy grande. Detengámonos, pues, un momento en ella para ver un poco más de cerca cómo se trata de superar, a partir de ella, el concepto clásico de identidad. De lo que hemos dicho se desprende que el eterno retorno de la diferencia se presenta, en realidad, como la ley de un mundo sin ser, sin unidad y sin identidad. Un mundo que es un mundo de diferencias, por lo que no supone ni lo uno ni lo mismo, sino que se construye sobre la caída del Dios único y sobre la ruina del yo idéntico... Un mundo, en suma, de fluctuaciones intensas, donde las identidades se pierden y donde cada uno no puede quererse más que queriendo también todas las demás posibilidades, haciéndose innumerables otros y aprendiéndose como un momento fortuito, cuyo azar mismo implica la necesidad de toda la serie entera. Pues bien, a este respecto Deleuze insiste en la diferencia de naturaleza que separa la copia y el simulacro para protestar contra su confusión como meras diferencias de grado. Porque la copia se define justamente por su grado de semejanza con el modelo, mientras que el simulacro se caracteriza por sus meros efectos externos de semejanza, lo cual no es lo mismo ni mucho menos. Y esto conduce a la evidencia de que el pensamiento postmetafísico es sustancialmente el pensamiento que acaba con esa maldición tradicional del simulacro al hacer de la semejanza el efecto externo del simulacro, y de la identidad la semejanza que se dice a partir de la afirmación de la diferencia entre series heterogéneas. O para decirlo más brevemente y más fácil de entender: Las cosas no son más que el simulacro mismo, mientras que el simulacro es la forma superior, puesto que lo difícil para toda cosa es alcanzar su propio simulacro.

El problema, por tanto, más importante del conocimiento de algo, de la realización de algo, no es otro que éste: ‘cómo una cosa, una obra de arte, una teoría científica, pero también, y sobre todo, un yo, alcanzan su propio simulacro? ¿Cuál es el proceso que permite a una significación formarse y desaparecer 
gracias a la producción del sentido y del no sentido? Según lo que venimos diciendo, solo se puede responder a esto diciendo que la identidad actual, por ejemplo, de un yo no sería otra cosa que la imagen del llegar a ser otro, o sea, la imagen de todas las diferentes identidades que le han precedido en otros diferentes estados anímicos de revelación, y que han sido necesariamente olvidadas. Y puesto que el ser del yo actual es un convertirse en otro, querer ser yo tiene que ser necesariamente querer convertirse en otro distinto a ese yo idéntico y estable de la memoria y del origen en el movimiento de la diferencia y de la repetición. Siguiendo a Klossovski, Deleuze observa que la coherencia del pensamiento así estrictamente pensado puro excluye incluso, en último término, al yo mismo en el momento en que se piensa. Por eso el yo ha de querer y afirmar el devenir y olvidarse de la identidad, pues el devenir le ofrece siempre de nuevo toda la serie de las identidades posibles, cuyo retorno solo se produce en el instante de la revelación.

Resumiendo, pues, toda esta temática de la diferencia y la repetición que Deleuze desarrolla en su espléndida obra que lleva este título y en la que se ve claramente la gran influencia de Nietzsche, podemos decir que la afirmación de la identidad del yo es simultánea a la afirmación del devenir. El yo renuncia a ser uno y el mismo de una vez por todas, y quiere la necesidad del movimiento circular, el eterno retorno. Al mismo tiempo, esta ruina de la noción clásica de sujeto como identidad estable es indisociable de la disolución del objeto como polo estable en la relación de conocimiento. Pues, para aparecer, la significación debe pasar necesariamente por todas las identidades posibles a otras significaciones que la harán retornar siempre la misma en el instante de la comprensión. De ahí la ilusión que supone la creencia en la estabilidad del referente y en la firmeza de lo que separa a lo verdadero de lo falso. El universo no es, en suma, más que un movimiento de continua huída de sí mismo, para reencontrarse de nuevo en la producción inagotable de lo múltiple y de lo diferente.

Pues bien, para terminar de ver la gran influencia del pensamiento de Nietzsche sobre la obra de Deleuze, nada mejor que proseguir este análisis en la significativa aplicación que Deleuze hace de este marco conceptual en la crítica al yo que lleva a cabo en El Antiedipo: Capitalismo y esquizofrenia (Deleuze y GuATTARI I 998). Una de las cosas que hacen de Deleuze un típico ejemplo de pensamiento estructuralista o postestructuralista o neoestructuralista, es su firme y total oposición a la tradicional concepción del yo como centro autosubsistente de actividad y de libertad, y a las múltiples versiones de ese principio en filosofías que eran coetáneas a Deleuze y con las que él debatió y dialogó, como eran la fenomenología, el existencialismo, el personalismo, el marxismo, y demás clases de humanismo. Aquello por lo que se define el estudio 
estructuralista de lo humano es que la lengua es siempre anterior respecto del hablante, el ello respecto del yo, la organización respecto del individuo, de modo que la estructura es lo que "pone en escena" al sujeto. O sea, el sujeto no es más que el lugar de encuentro de una serie de procesos que lo atraviesan determinándolo a ser lo que es, y hacen que, más que hablar él mismo, el sujeto "sea hablado", más que pensar, "sea pensado" o más que actuar, "sea actuado".

De modo que las tradicionales categorías de libertad, de acción, de conciencia, en lugar de explicar al hombre, confunden su naturaleza, y esto tanto desde el punto de vista ontológico (qué es el hombre), como desde el punto de vista gnoseológico-metodológico (cómo se conoce al hombre), representando graves obstáculos epistemológicos al desarrollo de una adecuada ciencia sobre lo humano. ¿Cómo se debería estudiar, pues, lo humano o al hombre? Pues el mejor modo de hacerlo debería ser, dice Deleuze, disolviéndolo, o sea, esforzándose por captar, más allá del yo y de sus presuntas facultades, la combinatoria anónima de leyes, procesos, estructuras y principios que lo gobiernan encubiertamente. "No se puede conocer nada de los hombres", es la famosa frase de Lévi-Strauss, si no es bajo la absoluta condición de deshacer el mito filosófico del hombre.

Es comprensible, pues, que esta nueva perspectiva de un pensamiento más allá del sujeto y en favor de una especie de antropología sin el hombre, discurran continuamente en abierta polémica con todas las filosofías que tienden a considerar el cogito o la conciencia como datos primarios e irreductibles de la condición humana. Para Deleuze, la conciencia no es más que el reflejo deformado y desconocido de los mecanismos inconscientes que la producen, y que no coincide nunca, por tanto, ni con toda la psique, ni con todo el hombre. Es más, siempre subyace y determina a la conciencia lo impensado, lo impensado, o sea toda esa serie de mecanismos extra-conscientes que se configuran como lo permanentemente otro de ella, y que escapan a la jurisdicción del pensamiento pensante. Por tanto, el límite teórico de las filosofías humanistas y metafísicas del yo consistiría en no haber visto, tras los avances de la lingüística y del psicoanálisis, que el individuo "está actuado" por una pluralidad de fuerzas de las cuales, no solo no es el sujeto, sino de las que tampoco es consciente. En concreto, el psicoanálisis nos enseña que la verdadera dimensión del hombre está siempre en otro lugar respecto a la consciencia y a las intenciones conscientes del sujeto.

Por ello, la gran importancia de la obra de Freud radica en que nos revela que el sujeto real, el individuo en su específica esencia, no tiene el aspecto de un yo centrado sobre la "conciencia" o la "existencia" o el "comportamiento", sino que está constitutivamente descentrado, constituido por una estructura que tiene un "centro" solamente en el desconocimiento imaginario del "yo", 
o sea, en las formaciones ideológicas en las cuales se "reconoce". Este reconocimiento de la trascendental aportación de Freud no significa, en cambio, que se pueda admitir su enfoque de la relación consciente-inconsciente en los términos en que él la propone. Hay que reconocer, ciertamente, a Freud el gran mérito, no solo de haber descubierto la importancia del inconsciente en el comportamiento humano, sino también la perspicacia de haberlo comprendido como productividad deseante ilimitada para la que todo es posible. Potencia deseante que no conoce otra ley que la del principio del placer, o sea, exigencia de satisfacción inmediata e incondicional. Entonces, lo que Deleuze critica a Freud es, justamente, haber retrocedido espantado ante este mundo de producción salvaje y de deseo explosivo que no reconoce ningún orden ni está dispuesto a someterse a nada. El gran fallo de Freud habría consistido, en suma, en haber desvanecido la importancia de ese descubrimiento al reducirlo al carácter y la estructura de un único deseo, el deseo sexual, y comprender su evolución a partir del complejo de Edipo como mecanismo esencial del dinamismo del inconsciente.

Y es que la primera y principal consecuencia de esta edipización del inconsciente es su carácter represivo y normalizador, la represión y amortiguamiento de su compleja y explosiva potencia deseante. Porque impone, en el origen mismo, el orden de la separación de las generaciones y de los sexos que es el fundamento de la identidad personal: yo "soy hijo de..., padre de..., esposo de..." Estas distinciones, que dan sentido a la estructura familiar, se convierten en el fundamento de la autoridad paterna en cuanto paradigma de la ley, de la alteridad inaccesible (el otro sexo) y de la finitud con todas sus delimitaciones fijadas ya de una manera esencial (el nacimiento y la muerte, yo y el otro, etcétera). De modo que quien acepta este orden es el individuo normal y moral y puede ser lo feliz que un hombre puede ser en una sociedad normalizada, mientras que quien no lo acepta queda fuera de la normalidad. Para Deleuze, por tanto, el sentido más propio de esta edipización consiste en que representa una verdadera "castración del deseo". Y ello por varias razones, pero, especialmente, porque implica una comprensión del deseo como algo negativo y culpable. Tendríamos que valorar el deseo no como algo de lo que tenemos que sentir miedo y avergonzarnos, sino como un impulso positivo, productivo, creador de realidad y de experiencias nuevas.

Lo que hace Freud con su edipización es convertirlo en carencia de un objeto ya existente y fijado por la naturaleza o la sociedad, objeto al que el deseo aspira pero que se mantiene inaccesible o prohibido (la madre). Cuando entendemos el deseo como algo positivo, en cambio, no es posible entender por deseo solo ese deseo unívoco y de sentido único que nunca significa otra cosa que el deseo sexual e incestuoso. No es admisible el argumento freudiano 
según el cual, a causa de su prohibición e imposibilidad, ese deseo original de la madre se manifiesta de distintos modos y va de objeto en objeto. En realidad, estas metamorfosis del deseo son algo más que solo máscaras y elaboraciones que transfieren y subliman el deseo edípico. Porque el verdadero deseo es múltiple y polisémico, libre de todo sentido común único e impuesto de antemano. Y la segunda consecuencia de esta edipización del deseo por parte de Freud no es menos debilitadora de su potencia positiva y creativa. Porque, para todo el psicoanálisis, el deseo edípico incestuoso es, de manera sustancial, culpable, de modo que la posible esperanza de curación psicoanalítica que los neuróticos, que somos todos, podemos esperar, se basaría en el reconocimiento de esta culpa y en la reafirmación de la ley que, en la infancia, todo el mundo ha transgredido inconscientemente, o sea, la ley de la prohibición del incesto con la que comienza y en la que se basa la sociedad humana.

Pues bien, ¿cómo se tiene que concretar ese arrepentimiento y ese propósito de la enmienda por haber sido incestuosos como forma de curarnos y superar nuestra neurosis? Respuesta: mediante la transferencia del deseo de nuestra madre a otra mujer distinta. Esto nos convierte entonces en padres y en continuadores y transmisores de la ley. Cualquiera puede advertir, sin mucho esfuerzo, el condicionamiento judío que estas ideas tienen en la obra de Freud, porque esta culpabilidad del deseo edípico hace pensar inequívocamente en el pecado original, lo que lleva a Deleuze a calificar a los psicoanalistas de "nuevos sacerdotes". Ellos son los nuevos curas a los que hay que ir a confesarse y a que nos den la absolución (Deleuze y Guattari 1998, 62 ss). En cualquier caso, esta culpabilidad inherente al deseo edípico es lo que produce que todo individuo edipizado esté siempre neurótico, e incluso paranoico, en función del grado en que la estructura edipiana le afecta en orden a identificarse más o menos con el papel de padre, legislador, juez, verdugo, autoridad, etcétera. Es, según Deleuze, lo mismo que siempre habrían hecho los sacerdotes cristianos. Crear el sentido y la conciencia del pecado y de la culpa en los individuos para obligar a confesarnos y obtener así de nosotros beneficios de diverso tipo.

Pues bien, todo el discurso sobre la esquizofrenia que contiene El Antiedipo en su capítulo IV es el modo en el que Deleuze intenta llevar a cabo su crítica a esta edipización freudiana del deseo, y que no podemos ya desarrollar ni analizar aquí. Solo decir que el esquizofrénico es, en este planteamiento crítico, la imagen del individuo que se resiste a la edipización ordinaria y a todo psicoanálisis edipizante. Naturalmente por ello, su delirio no fijable, incontrolable y radicalmente no significativo lo excluye del campo psicoanalítico y de la sociedad que lo excluye como enfermo. Porque, de hecho, como habrá podido advertirse ya, el freudiano psicoanálisis edipiano está claramente concebido para que funcione al servicio de la represión social: es su producto y 
su colaborador, como antes lo había sido la religión cristiana. Pues resolver el complejo de Edipo no significa otra cosa que interiorizar la culpa, o sea, la ley (del padre, pero también y sobre todo de la sociedad), respetarla, hacerla respetar y transmitirla a los hijos. Lo que Freud expresa, pues, con su edipización del deseo es que la represión social es, en todo caso, inevitable, culpabilizadora y primordial.

Por ello, el esquizoanálisis es la alternativa crítico-paródica que opone Deleuze al psicoanálisis freudiano. El objetivo de este esquizoanálisis sería llevar a cabo, justamente y de manera experimental, una desedipización del inconsciente, tanto en el individuo como en la sociedad con consecuencias interesantes desde una perspectiva política y revolucionaria. Al igual que Foucault, Deleuze considera a la sociedad, y a la familia que la sintetiza, como los agentes que convierten al esquizofrénico en un enfermo. Pero la locura no es, la mayoría de las veces, más que una construcción sociopolítica porque, como tal, el esquizofrénico no es sino alguien que reacciona y se resiste a la edipización normalizadora. El esquizofrénico no es, aquí, más que el individuo que confunde todos los códigos, en particular el binario, porque está a favor de lo múltiple y no de lo dual. O sea, no esclavizado por la identidad, sino que se entrega, "en un deslizamiento rápido", al libre juego de las identificaciones simbólicas transitorias y simultáneas: "Yo, Antonin Artaud, soy mi hijo, mi padre, mi madre y yo, hombre y mujer, padre e hijo, muerto y vivo": "El esquizo — dice Deleuze- no (re)conoce al sujeto, o sea, no tiene 'yo' fijo, pues el yo solo se fija con el Edipo. Los goces y los sufrimientos infinitos del deseo-delirio tienen esta no fijación como precio" (Deleuze y Guattari i 998, 249).

En suma, mientras el psicoanálisis apunta a que uno construya su yo, el esquizoanálisis invita a encontrar el cuerpo propio y a deshacer el yo del pensamiento-escritura rizomático. Se trata, en último término, de invertir lo negativo del nihilismo reactivo para ver en él algo positivo y creativo subrayando las diferencias afirmativas, es decir, las diferencias por las que un ser, un acontecimiento o un valor se afirman de una manera creadora por sí mismos, mientras que las diferencias negativas o reactivas solo apuntarían a distinguirse del otro y a oponerse al otro. 


\section{BiBLIOGRAFÍA}

Deleuze, G. I962, Nietzsche et la philosophie, París: PUF.

Deleuze, G. I964, Marcel Proust et les signes, París: PUF.

Deleuze, G. I966, Le Bergsonisme, París: PUF.

Deleuze, G. I969, Logique du sens, París: Minuit.

Deleuze, G. 2002, Diferencia y repetición, trad. M. Silvia, Buenos Aires: Amorrortu.

Deleuze, G. y Guattari, F. i998, El Antiedipo: Capitalismo y esquizofrenia, trad. F. Monge, Barcelona: Paidós.

Nietzsche, F. 2006-20io, Fragmentos Póstumos, D. Sánchez Meca (ed.), trad. J. L. Vermal, Madrid: Tecnos, 4 vols.

Nietzsche, F. 20 io ss, Obras Completas, D. Sánchez Meca (ed.), trad. Joan B. Llinares, Luis E. de Santiago, Alejandro Martín, Juan L. Vermal, M. Barrios, M. Parmeggiani, J. Aspiunza, D. Sánchez Meca, Madrid: Tecnos, 4 vols. 